\title{
Evolutionary forces in Mycobacterium tuberculosis: implications for product development
}

\author{
Sebastien Gagneux ${ }^{1,2,3}$ \\ From Immunodiagnosis of Tuberculosis: New Questions, New Tools \\ Virginia, VA, USA. 21-23 September 2008
}

Research on the evolutionary forces that have shaped the genetic diversity in Mycobacterium tuberculosis (M.tb), in particular natural selection, random genetic drift, migration, and changes in demography have important implications for product development. Until now, scientists have not been able to quantify the genetic differences among M.tb groups. By tracking genomic deletions, six major lineages of M.tb have been identified in humans, with distinct geographical distributions. The Asian M.tb lineages cause the highest global TB burden, but most research has been done on EuroAmerican strains. Our team has performed a multilocus sequencing analysis of 89 genes in 99 strains of human M.tb, eight strains of animal-adapted strains and the distantly-related M. canettii. We have worked to identify the sources of diversity in human-adapted M.tb and the evolutionary forces that shape this diversity. Using the DNA sequences of the 89 selected genes as a basis for a comparison, then we constructed a phylogentic tree which supported the M.tb groupings defined earlier by genomic deletion analyses. However, using DNA sequence data, actual genetic distances could be computed that suggested there was as much difference between some human strains as between $\mathrm{M}$. bovis and some human M.tb strains. Our research has also determined that the six human-adapted M.tb lineages are part of two main clades, an evolutionary "ancient" clade found in the Philippines, around the Indian Ocean, and in West Africa, and an evolutionary "modern" clade with a much wider geographic distribution.

Additionally, the role of selection in M.tb evolution was analyzed through the ratio per site of non-synonymous nucleotide substitutions $(\mathrm{dN})$, which change the amino acid sequence, to synonymous nucleotide substitutions $(\mathrm{dS})$ that do not result in a change in the amino acid sequence $(\mathrm{dN} / \mathrm{dS})$. The $\mathrm{dN} / \mathrm{dS}$ in M.tb was 0.57, much higher than most other bacteria. We explored three possible reasons for this high $\mathrm{dN} / \mathrm{dS}: 1$ ) M.tb is a recently-evolved pathogen and insufficient time has occurred for natural selection to remove nonsynonymous single nucleotide polymorphism (nSNP) mutations which are likely to be deleterious, 2) the host immune system is selecting for variability in M.tb to evade the host immune responses, or 3) natural selection against nSNPs is reduced. Our team concluded that the latter explanation was the most likely as we found that $58 \%$ of non-synonymous changes in M.tb occurred in regions of proteins that were highly conserved in other mycobacterial species. This suggests that purifying selection against nSNPs is reduced in M.tb compared to other bacteria, and as a consequence many of these nSNPs are likely to impact protein function.

\section{Author details}

'Swiss Tropical and Public Health Institute, 4051 Basel, Switzerland ${ }^{2}$ University of Basel, 4003 Basel, Switzerland. 'Medical Research Council, National Institute for Medical Research, London NW7 1AA,UK.

Published: 17 December 2010

doi:10.1186/1753-6561-4-S3-O4

Cite this article as: Gagneux: Evolutionary forces in Mycobacterium tuberculosis: implications for product development. BMC Proceedings 2010 4(Suppl 3):O4. 